\title{
Assessment of acute pancreatitis severity via determination of serum levels of hsa-miR-126-5p and IL-6
}

\author{
YING-JIE CHEN $^{1 *}$, TIAN-LAI LIN ${ }^{2 *}$, ZHE CAI $^{3}$, CHANG-HU YAN $^{1}$, SEN-REN GOU ${ }^{1}$ and YAO-DONG ZHUANG ${ }^{1}$ \\ ${ }^{1}$ Department of Critical Care Medicine, Jinjiang Hospital of Traditional Chinese Medicine, Jinjiang, Fujian 362200; \\ ${ }^{2}$ Department of Critical Care Medicine, Quanzhou First Hospital Affiliated to Fujian Medicine University, \\ Quanzhou, Fujian 362000; ${ }^{3}$ Department of Emergency Medicine, \\ Jinjiang Hospital of Traditional Chinese Medicine, Jinjiang, Fujian 362200, P.R. China
}

Received September 19, 2019; Accepted July 23, 2020

DOI: $10.3892 / \mathrm{etm} .2020 .9458$

\begin{abstract}
Early assessment of acute pancreatitis (AP) severity is key to its treatment. The present study aimed to explore the role of microRNAs (miRNAs/miRs) combined with inflammatory factors in determining AP severity. For this, serum pro-inflammatory cytokines [tumor necrosis factor (TNF)- $\alpha$, interleukin (IL)-1, IL-6, IL-8 and IL-10)] and miRNAs [Homo sapiens (hsa)-miR-548d-5p, hsa-miR-126-5p and hsa-miR-130b-5p] were detected in patients with mild AP (MAP), severe AP (SAP) and recurrent AP (RAP). High expression of IL-10, TNF- $\alpha$, hsa-miR-126-5p, hsa-miR-548d-5p and hsa-miR-130b-5p was able to distinguish SAP from MAP and RAP $(\mathrm{P}<0.05)$. Multifactorial binary logistic regression analysis indicated that IL-1/IL-6 combined with hsa-miR-126-5p/hsa-miR-548d-5p had a significant influence on AP and AP severity $(\mathrm{P}<0.05)$. Receiver operating characteristic analysis revealed that IL-1 combined with hsa-miR-126-5p [area under the curve (AUC), 0.926; sensitivity, 90.0\%; specificity, $86.7 \%, \mathrm{P}<0.001]$ and IL-6 combined with hsa-miR-126-5p (AUC, 0.952; sensitivity, 93.3\%; specificity, $90.0 \%$; $\mathrm{P}<0.001$ ) were able to better distinguish MAP from SAP than IL-1/IL-6 combined with hsa-miR-548d-5p, lipase, and amylase. IL-1 or IL-6 combined with hsa-miR-548d-5p (AUC, 0.924; sensitivity, 83.3\%; specificity, 93.3\%; P<0.001) were able to better distinguish SAP from RAP than IL-1/IL-6 combined with hsa-miR-126-5p, lipase, and amylase. IL-1
\end{abstract}

Correspondence to: Dr Ying-Jie Chen, Department of Critical Care Medicine, Jinjiang Hospital of Traditional Chinese Medicine, 1105 Quan'an Middle Road, Jinjiang, Fujian 362200, P.R. China

E-mail: chenyj@fjtcm.edu.cn

${ }^{*}$ Contributed equally

Abbreviations: AP, acute pancreatitis; MAP, mild AP; SAP, severe AP; RAP, recurrent AP; AUC, area under the ROC curve; ROC, receiver operating characteristic, RT-PCR, reverse transcription PC

Key words: acute pancreatitis, microRNA, inflammatory cytokines, predictors, receiver operating characteristic curve combined with hsa-miR-126-5p (AUC, 0.926; sensitivity, 90.0\%; specificity, $86.7 \%$; $\mathrm{P}<0.001)$ and IL-6 combined with hsa-miR-126-5p (AUC, 0.952; sensitivity, 93.3\%; specificity, $90.0 \% ; \mathrm{P}<0.001)$ were able to better differentiate between MAP and RAP than IL-1/IL-6 combined with hsa-miR-548d-5p, lipase, and amylase. These results demonstrated that the combined detection of serum IL-6 and hsa-miR-126-5p may be useful for the early prediction of AP classification.

\section{Introduction}

Acute pancreatitis (AP) is the activation of pancreatic enzymes followed by local inflammation of the pancreas and systemic inflammatory response syndrome, which is the major characteristic of the disease. AP may be divided into three categories: Mild AP (MAP) refers to AP that is not accompanied by organ failure or local or systemic complications. Moderately severe AP (MSAP) is accompanied by transient organ failure $(>48 \mathrm{~h})$ or local or systemic complications. Severe AP (SAP) indicates AP with persistent organ failure $(>48 \mathrm{~h})(1,2)$. The major difference between MSAP and SAP is the duration of organ dysfunction. Recurrent AP (RAP) refers to the presence of two or more confirmed episodes of AP after which pancreatic tissue and function return to normal. The treatment methods for MAP, SAP and RAP are different (3). Thus, suitable indicators to distinguish among MAP, SAP and RAP should be determined. Determining the severity of AP early is important to select the appropriate treatment and determine the prognosis. Although the Acute Physiology and Chronic Health Evaluation II (APACHE II) and Ranson scoring systems may be used to predict the severity of AP, they are relatively complex (4). Therefore, simple and quick clinical signs and serum indicators to accurately predict disease severity may be beneficial.

Tumour necrosis factor (TNF)- $\alpha$, interleukin (IL)-1 $\beta$, IL- 6 , IL-8 and IL-10 are important inflammatory cytokines markers in the inflammatory development of AP. In a previous study, the changes in monocyte chemoattractant protein (MCP)-1, TNF- $\alpha$ and IL- 8 in the serum of the MAP group were different from those of the MSAP and SAP groups, suggesting that the levels of MCP-1, TNF- $\alpha$ and IL- 8 are associated with the severity of AP and may serve as diagnostic indicators (5). 
Severe forms of AP are associated with increased serum levels of IL-6, IL-10 and IL-1 (6). IL-6 is a significant indicator of AP severity (7). These studies suggest that inflammatory cytokines have an important role in the severity of AP.

MicroRNAs (miRNAs/miRs) are endogenous noncoding regulatory single-stranded small molecule RNAs, the length of which is approximately $18-25$ nucleotides. RNAs and miRNAs have an important role in the regulation of gene expression and abnormal expression of miRNAs may increase the incidence of AP (8). The role of miRNAs in AP is an increasing area of study. Homo sapiens (hsa)-miR-10b and hsa-miR-146a in the peripheral blood of patients with AP have been indicated to be a predictor of disease severity (9). It has also been reported that hsa-miR-126a-5p and hsa-miR-551b-5p are able to predict AP severity (MAP and SAP) (10). Through targeting multiple factors of crucial pathways, hsa-miR-548d-5p is considered to be a possible superior regulator of the development of pancreatic cancer (11). Overexpression of miR-130b markedly reduced the invasive ability of pancreatic cancer cells by targeting STAT3 (12). However, the association between miRNAs and AP has remained largely elusive.

The early prediction of AP severity is important for treatment. To date, the existing indicators are not widely used in clinics. Certain studies have reported that levels of cytokines (e.g., TNF- $\alpha$, IL-6 and IL-8) and miRNAs (e.g., hsa-miR-551b-5p, hsa-miR-126-5p and hsa-miR-146a) may be used to distinguish between different levels of AP severity. Severe forms of AP were associated with the levels of IL-6, IL-10 and IL-1 receptor antagonist in serum (5-10). However, whether miRNA-binding inflammatory factors are able to predict AP severity has remained to be determined. Therefore, the aim of the present study was to explore the value of miRNAs (hsa-miR-548d-5p, hsa-miR-126-5p and hsa-miR-130b-5p) combined with inflammatory cytokines (TNF- $\alpha$, IL-1, IL-6, IL-8 and IL-10) in predicting the severity of AP in order to provide laboratory indicators for the early prediction of AP severity.

\section{Materials and methods}

Human subjects. A total of 90 patients with AP who were admitted to the Department of Critical Care Medicine of Jinjiang Hospital of Traditional Chinese Medicine (Jinjiang, China) between May 2017 and January 2019 were included in this study. AP was defined as characteristic abdominal pain with a threefold increase in levels of serum lipase or amylase. The severity of AP was determined on the basis of the Revised Atlanta Classification of AP (2012) (1). In the present study, patients with clinical symptoms of acute abdominal pain and vomiting who had signs of peritonitis, such as abdominal distension, lumbar rib tenderness and swelling, suggested abdominal exudation, inflammation and intraperitoneal hypertension were enrolled. Blood samples were collected from all patients within $24 \mathrm{~h}$ of admission, and serum amylase and lipase activities were measured. The reference value of amylase was 25-125 U/1 (13) and the reference value of lipase was 23-300 U/1 (14). Abdominal CT scan was used as the standard imaging method and enhanced CT scan was performed on patients with an indefinite diagnosis (Fig. 1). The severity of AP was determined according to the Balthazar CT scoring system (15). MAP, SAP and RAP patients of similar age, sex and etiology were screened. There were 30 cases in each group. The AP group consisted of 36 females and 54 males aged between 24 and 72 years with a mean age of $46.91 \pm 9.75$ years. The exclusion criteria were an age of $<18$ years, pregnancy or breastfeeding, liver or kidney failure, and malignancy, or presentation at $>24 \mathrm{~h}$ after the onset of symptoms. Patients who did not agree to provide written informed consent were also excluded.

As the normal control (NC) group, 30 healthy individuals presenting in the same time period, sex and age-matched to the cases were selected. They consisted of 15 females and 15 males aged from 29 to 71 years with a mean age of $46.56 \pm 11.05$ years. The healthy controls were recruited at the Physical Examination Center of Jinjiang Hospital of Traditional Chinese Medicine (Jinjiang, China). The reasons for NC group patients receiving CT scans included cholecystitis, gallbladder polyps and gallstones. The exclusion criteria were as follows: Any systemic and chronic diseases, such as autoimmune diseases, tumour and diabetes, or cardiovascular diseases, such as hypertension, coronary artery disease and hyperlipidemia. The study was approved by the ethics committee of Jinjiang Hospital of Traditional Chinese Medicine (Jinjiang, China).

Sample collection. Venous blood $(10 \mathrm{ml})$ was extracted from the patients prior to treatment. Samples were collected from the patients' cubital vein using Monovette tubes (Sarstedt). Serum was separated by centrifugation $(1,000 \mathrm{x} \mathrm{g,} 5 \mathrm{~min}$, room temperature) and stored at $-80^{\circ} \mathrm{C}$ until analysis. As haemolysis may falsely indicate increases in either inflammatory cytokines or miRNA levels $(16,17)$, each visually detected haemolysed serum sample was rejected. In the present study, all blood samples were processed and screened by the hospital's experienced laboratory physician. As exemplified in Fig. 2, samples 1 and 2 were judged as hemolyzed samples and discarded, while samples 3,4 and 5 were determined to be non-hemolytic samples and used for subsequent testing.

Detection of the levels of inflammatory factors (TNF- $\alpha, I L-1$, IL-6, IL-8 and IL-10) in serum by ELISA kits. Arterial blood $(5 \mathrm{ml})$ was extracted from the patients prior to treatment. The serum was centrifuged at $1,000 \mathrm{x}$ g for $5 \mathrm{~min}$. The levels of TNF- $\alpha$, IL-1, IL-6, IL- 8 and IL-10 in serum were measured by ELISA. TNF- $\alpha$, IL-1, IL-6, IL- 8 and IL-10 kits were all purchased from Shanghai Novus Biotechnology Co., Ltd. The ELISA kit details were as follows: Human TNF- $\alpha$ ELISA kit (colorimetric), cat. no. NBP1-91170; human IL-1 $\alpha / \mathrm{IL}-1 \mathrm{~F} 1$ ELISA kit (colorimetric), cat. no. NBP1-91187; human IL-6 ELISA kit (colorimetric), cat. no. NBP1-89869; human CXCL8/IL-8 ELISA kit (colorimetric), cat. no. NBP1-83743; human IL-10 ELISA Kit (colorimetric), cat. no. NBP1-89871. All procedures were performed according to the manufacturer's instructions. The optical density values were read by a microplate reader (wavelength, $450 \mathrm{~nm}$ ), and the corresponding concentrations of TNF- $\alpha$, IL-1, IL-6, IL-8 and IL-10 were calculated and recorded.

Quantification of miRNAs by reverse transcription-quantitative $(R T-q) P C R$. After thawing, serum samples were 

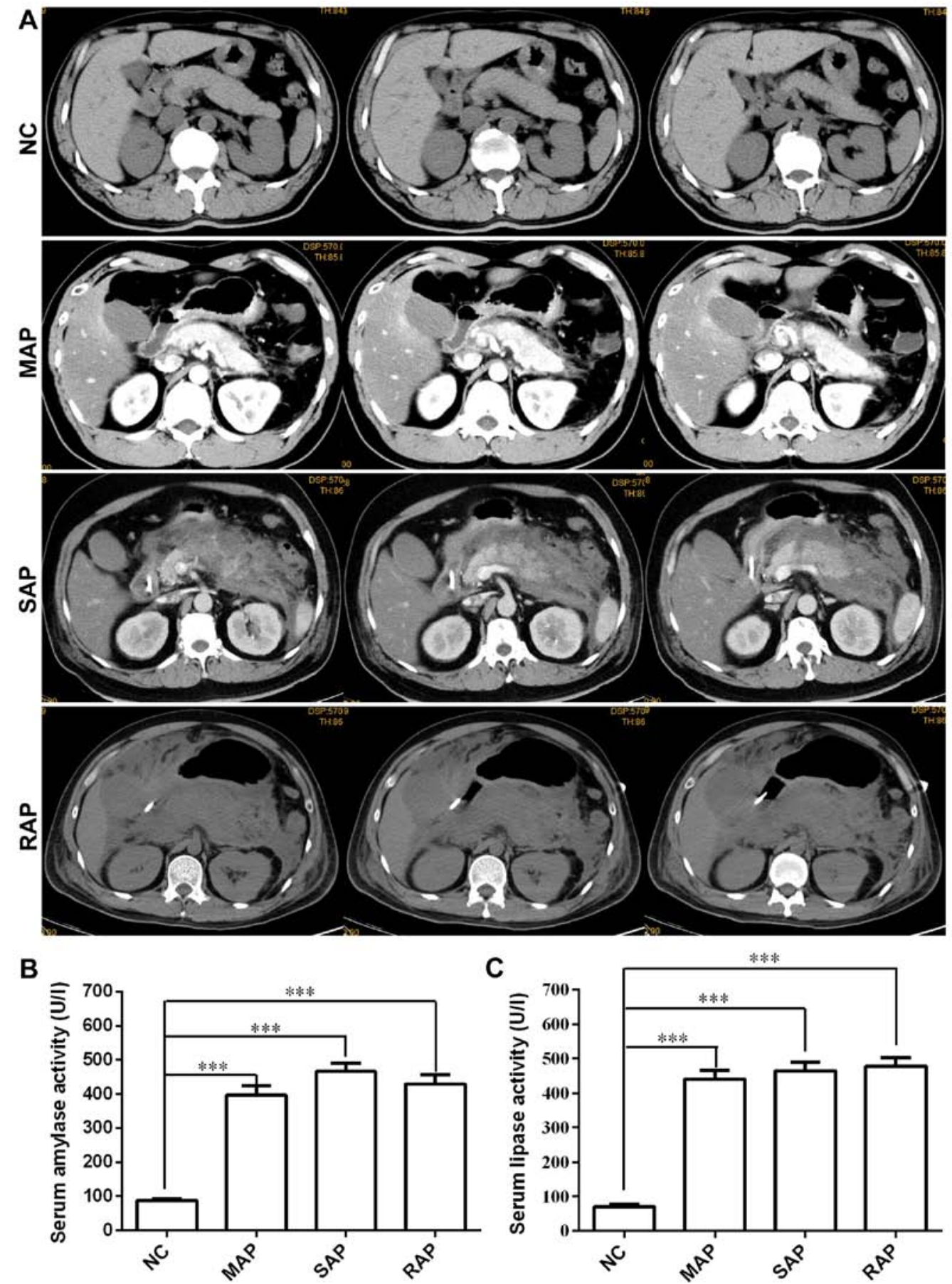

Figure 1. Clinical manifestations of patients with different severities of AP. (A) CT or enhanced CT images of the patients with pancreatitis. NC, normal pancreatic structure; MAP, enhanced scanning shows uneven signal enhancement, pancreatic oedema and exudation; SAP, a large number of flocs exudate around the pancreas and accumulate into inflammatory masses or diffuse extended inflammation; RAP, diffuse enlargement and vague margins of the pancreas, visible strips and flocculent exudation in the fat space around the pancreas, and bilateral pleural effusion. (B) Serum amylase and (C) lipase activity in patients with acute pancreatitis was significantly increased. Values are expressed as the mean \pm standard error. One-way analysis of variance with Dunnett's test was used for assessment of intergroup differences. ${ }^{* * *} \mathrm{P}<0.001$. Groups: $\mathrm{NC}$, normal control; MAP, mild AP; SAP, severe AP; RAP, recurrent AP; AP, acute pancreatitis.

centrifuged at room temperature and $1,000 \mathrm{x}$ g for $5 \mathrm{~min}$ to pellet any debris and insoluble components. Circulating miRNA was extracted using the miRCURY RNA Isolation kit (Exiqon) according to the manufacturer's protocol. The purified miRNA was eluted with $20 \mu 1$ RNase-free water. The isolated miRNAs were assessed by an Agilent Bioanalyzer 2100 system (Agilent Technologies, Inc.). Total RNA was isolated from the serum samples and treated with RNase-free water according to the NucleoZOL ${ }^{\circledR}$
(Gene Co., Ltd.) method. Primers were resuspended by adding $250 \mu 1 \mathrm{RNase}$-free water. Master mix was prepared for each miRNA-specific assay. Each single reaction included $10 \mu \mathrm{l}$ of qPCR SYBR ${ }^{\circledR}$-Green Master Mix (Promega Corporation), $10 \mu \mathrm{l}(10 \mu \mathrm{M})$ of primer set for an individual miRNA, $3 \mu \mathrm{l}$ of RNase-free water and $2 \mu 1$ (20 ng) of RT product added to a single real-time PCR tube as a template.

First, $0.5 \mu \mathrm{g}$ total RNA was reverse transcribed using the Reverse Transcription System kit (Takara Bio, Inc.). The 
Table I. Primers for RT and qPCR.

\begin{tabular}{lll}
\hline Gene/miRNA & & \multicolumn{1}{c}{ Primer sequence (5'-3') } \\
\hline hsa-miR-130b-5p & F & CGCGACTCTTTCCCTGTTG \\
& R & AGTGCAGGGTCCGAGGTATT \\
RT & GTCGTATCCAGTGCAGGGTCCGAGGTATTCGCACTGGATACGACGTAGTG \\
hsa-miR-126-5p & F & GCGCGCATTATTACTTTTGG \\
& R & AGTGCAGGGTCCGAGGTATT \\
hsa-miR-548d-5p & RT & GTCGTATCCAGTGCAGGGTCCGAGGTATTCGCACTGGATACGACCGCGTA \\
& F & GCGCGAAAAGTAATTGTGGTT \\
& R & AGTGCAGGGTCCGAGGTATT \\
hsa-miR-103a-3p & RT & GTCGTATCCAGTGCAGGGTCCGAGGTATTCGCACTGGATACGACGGCAAA \\
& F & GCGAGCAGCATTGTACAGGG \\
& R & AGTGCAGGGTCCGAGGTATT \\
& RT & GTCGTATCCAGTGCAGGGTCCGAGGTATTCGCACTGGATACGACTCATAG
\end{tabular}

RT, reverse transcription; F, forward; R, reverse; miRNA/miR, microRNA; hsa, Homo sapiens.

(1)

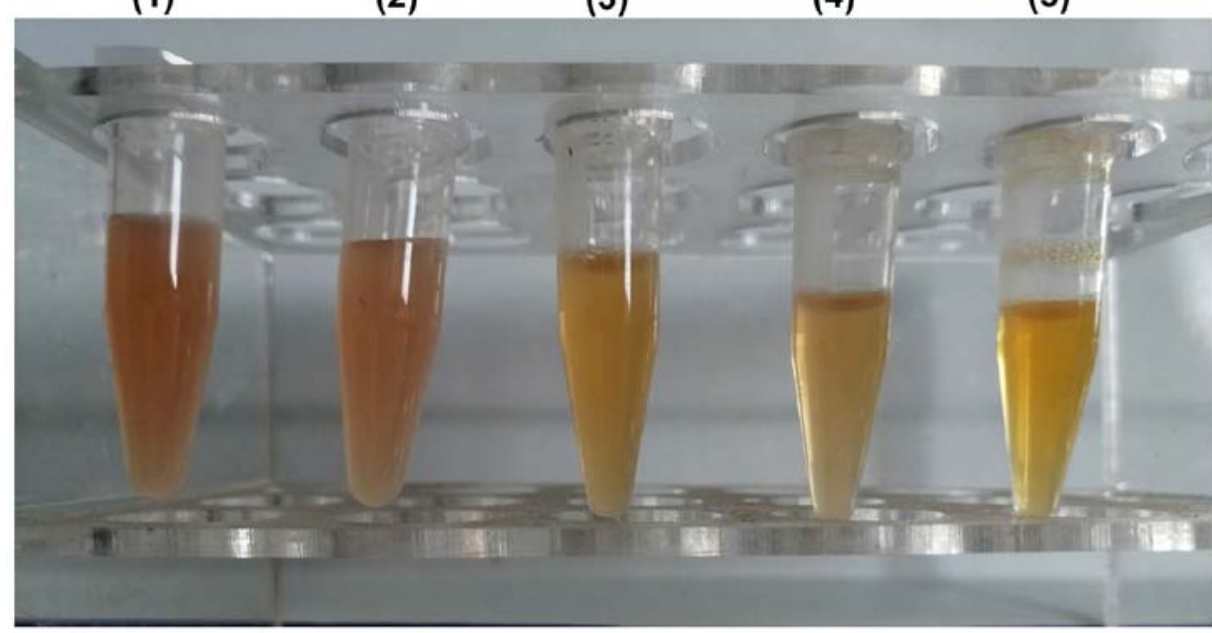

Figure 2. Screening of serum samples. In the present study, all blood samples were processed and screened by experienced laboratory physicians. (1 and 2) Serum samples were judged as hemolyzed samples and discarded; (3-5) Serum samples were determined to be non-hemolytic samples and used for subsequent testing.

synthesized complementary (c)DNA was amplified by qPCR using a Heal Force system [Heal Force; Likang Biomedical Technology Holdings Co., Ltd.; Lixin Instrument (Shanghai) Co., Ltd.] The reaction conditions for the RT step were $30^{\circ} \mathrm{C}$ for $10 \mathrm{~min}, 42^{\circ} \mathrm{C}$ for $50 \mathrm{~min}$ and $85^{\circ} \mathrm{C}$ for $10 \mathrm{~min}$. The resultant cDNA was used as a template for subsequent PCR. A total of 40 cycles of PCR amplification were performed, with initial incubation at $95^{\circ} \mathrm{C}$ for $5 \mathrm{~min}$ and final extension at $72^{\circ} \mathrm{C}$ for $5 \mathrm{~min}$. Each cycle comprised denaturation at $95^{\circ} \mathrm{C}$ for $10 \mathrm{sec}$, annealing at $60^{\circ} \mathrm{C}$ for $30 \mathrm{sec}$ and extension at $72^{\circ} \mathrm{C}$ for $30 \mathrm{sec}$. The miRNA primers used for RT-PCR amplification are presented in Table I. The mRNA expression level was normalized to that of hsa-miR-103a-3p (10). The relative expression of candidate genes was calculated using the formula $\Delta \Delta \mathrm{Cq}=\left(\mathrm{C}_{\mathrm{q} \text {, target }}-\mathrm{C}_{\mathrm{q}, \text { miR-103a-3p }}\right)_{\mathrm{AP}}-\left(\mathrm{C}_{\mathrm{q} \text {, target }}-\mathrm{C}_{\mathrm{q}, \text { miR-103a-3p }}\right)_{\mathrm{NC}}$, and the estimated expression ratio was equal to $2^{-\Delta \Delta \mathrm{Cq}}(18)$.

Statistical analysis. All data were analysed using SPSS 25.0 software (IBM Corp.). The Shapiro-Wilk test was used to analyze normality. If the data showed a normal distribution, one-way analysis of variance with Dunnett's test was used. If the data did not obey normal distribution, Mann-Whitney U test was used to compare the differences between two groups, and the Kruskal-Wallis test with Dunn's post-hoc test was applied to compare between multiple groups. Spearman's rank correlation analysis was used to analyse the correlation between inflammatory factors, miRNAs and AP. Univariate binary classification logistic regression analysis was used to determine the association between the clinical condition of patients with AP and the indicators assessed. Multivariate logistic regression analysis was used to analyse the influence of the indexes tested on MAP, SAP and RAP. Receiver operating characteristic (ROC) analysis was used to determine the clinical accuracy of significant serum pro-inflammatory factors and miRNAs, and the area under the ROC curve (AUC) was calculated for each miRNA of interest using DeLong's method. $\mathrm{P}<0.05$ was considered to indicate statistical significance. 
Table II. General characteristics of the patients.

\begin{tabular}{|c|c|c|c|c|c|c|}
\hline Factor & $\begin{array}{c}\text { AP } \\
n=90 \text { case }\end{array}$ & $\begin{array}{c}\text { MAP } \\
n=30 \text { case }\end{array}$ & $\begin{array}{c}\text { SAP } \\
\mathrm{n}=30 \text { case }\end{array}$ & $\begin{array}{c}\text { RAP } \\
n=30 \text { case }\end{array}$ & $\begin{array}{c}\mathrm{NC} \\
\mathrm{n}=30 \text { case }\end{array}$ & P-value \\
\hline Sex & & & & & & $0.86^{\mathrm{a}}$ \\
\hline Female & $36(40)$ & $13(43.33)$ & $11(36.67)$ & $12(40)$ & $15(50)$ & \\
\hline Male & $54(60)$ & $17(56.67)$ & $19(63.33)$ & $18(60)$ & $15(50)$ & \\
\hline Age (years) & 47 (IQR, 39-54) & $46(\mathrm{IQR}, 39-54)$ & 47 (IQR, 38-56) & 47 (IQR, 40-55) & 48 (IQR, 38-56) & $0.94^{\mathrm{b}}$ \\
\hline \multicolumn{7}{|l|}{ Etiology } \\
\hline Biliary disease & $45(50)$ & $16(53.33)$ & $18(60)$ & $11(36.67)$ & - & $0.33^{\mathrm{a}}$ \\
\hline Alcoholism & $27(30)$ & $8(26.67)$ & $7(23.33)$ & $12(40)$ & - & $0.53^{\mathrm{a}}$ \\
\hline Hypertriglyceridemia & $8(8.89)$ & $3(10)$ & $3(10)$ & $2(6.67)$ & - & $0.95^{\mathrm{a}}$ \\
\hline Other & $10(11.11)$ & $3(10)$ & $2(6.67)$ & $5(16.67)$ & - & $0.67^{\mathrm{a}}$ \\
\hline Bathazar CT score & 2.5 (IQR, 1-3) & 1 (IQR, 1-2) & 3 (IQR, 3-4) & 2.5 (IQR, 2-3.25) & $0.5(\mathrm{IQR}, 0-1)$ & $<0.01^{\mathrm{b}}$ \\
\hline Smoking history & $35(38.89)$ & $7(23.33)$ & $13(43.33)$ & $15(50)$ & $13(43.33)$ & $0.28^{\mathrm{a}}$ \\
\hline Lipase (U/l) & $430.59 \pm 15.18$ & $396.46 \pm 26.71$ & $465.72 \pm 25.40$ & $429.59 \pm 26.08$ & $87.67 \pm 4.48$ & $<0.01^{\mathrm{c}}$ \\
\hline Amylase (U/l) & $460.67 \pm 14.41$ & $440.09 \pm 25.36$ & $463.96 \pm 25.80$ & $477.96 \pm 24.05$ & $70.27 \pm 6.56$ & $<0.01^{\mathrm{c}}$ \\
\hline
\end{tabular}

${ }^{\mathrm{a}} \mathrm{Chi}$-squared test; ${ }^{\mathrm{b}} \mathrm{Kruskal}-$ Wallis test; ${ }^{\mathrm{c}} \mathrm{One}$-way analysis of variance with Dunnett's test. The reference value of amylase was $25-125 \mathrm{U} / \mathrm{l}$ (13) and the lipase was 23-300 U/l (14). Values are expressed as n (\%), median with interquartile range or mean \pm standard error. Groups: SAP, severe AP; MAP, mild AP; RAP, recurrent AP. AP, acute pancreatitis.

\section{Results}

Characteristics of participants. Clinical data for the AP and NC groups are presented in Table II. A total of 90 patients with $\mathrm{AP}$ and 30 control cases were recruited into the study. The AP group was $60 \%$ male with a median age of 47 (interquartile range, 39-54) years, and $38.89 \%$ patients with a history of smoking. The NC group was 50\% male with a median age of 48 (interquartile range, $38-56$ ) years, and $43.33 \%$ patients had a history of smoking. There were no significant differences in sex, age or smoking status between the AP and NC group $(\mathrm{P}=0.34, \mathrm{P}=0.64$ and $\mathrm{P}=0.67$, respectively). Biliary disease was the most common etiology of pancreatitis, identified in 45 patients (50\%), followed by alcoholism (30\%) and hypertriglyceridemia (8.89\%). AP patients had a median Bathazar CT score of 2.5 (interquartile range, 1-3), and lipase levels of $430.59 \pm 15.18 \mathrm{U} / 1$ and amylase levels of $460.67 \pm 14.41 \mathrm{U} / 1$, which were higher than those in the $\mathrm{NC}$ group $(\mathrm{P}<0.01$, respectively).

Of the 90 AP patients, 30 cases were RAP patients. Based on the Revised Atlanta Classification of AP (1), the remaining patients were classified into the MAP and SAP group, 30 cases per group. The MAP group was $56.67 \%$ male, the median age was 46 (interquartile range, 39-54) years and $23.33 \%$ patients had a history of smoking. The SAP group was $63.33 \%$ male with a median age of 47 (interquartile range, 38-56) years and $43.33 \%$ of patients had a history of smoking. The RAP group was $60 \%$ male, the median age was 47 (interquartile range, 40-55) years, and $50 \%$ of patients had a history of smoking. The sex, age and smoking status between the AP, MAP, SAP, RAP and $\mathrm{NC}$ groups were not significantly different $(\mathrm{P}=0.86, \mathrm{P}=0.94$, $\mathrm{P}=0.28$, respectively). Biliary disease was the most common etiology of MAP (53.33\%) and SAP (60\%), followed by alcoholism (MAP, 26.67\%; SAP, 23.33\%) and hypertriglyceridemia
(10\% of each). In RAP patients, alcoholism (40\%) was the most common etiology, followed by biliary disease (36.67\%) and hypertriglyceridemia (6.67\%). There were no significant differences in etiologies between the MAP, SAP and RAP groups $(\mathrm{P}>0.05)$. The Bathazar CT score, lipase and amylase level in the AP, MAP, SAP and RAP groups were significantly increased compared to those in the NC group $(\mathrm{P}<0.01)$.

Levels of pro-inflammatory factors and miRNAs in the AP groups compared with the NC group. Comparison of the results with the nonparametric Mann-Whitney U test indicated that pro-inflammatory factors and miRNAs were significantly increased in the AP vs. the NC group $(\mathrm{P}<0.001$; Table SI).

Pro-inflammatory factors and miRNAs in patients with different classifications of AP. The levels of pro-inflammatory factors among the NC, MAP, SAP and RAP groups were compared (Fig. 3A-E). The results showed that IL-1 and IL-6 expression in the MAP, SAP and RAP groups was significantly higher than that in $\mathrm{NC}$ group $(\mathrm{P}<0.001)$. Compared with NC group, the expression of IL-8, IL-10 and TNF- $\alpha$ in the SAP group was significantly increased $(\mathrm{P}<0.001)$, and IL-10 was also significantly increased in the RAP group when compared with the NC group $(\mathrm{P}<0.05)$. The levels of IL-8, IL-10 and TNF- $\alpha$ in the MAP group were significantly lower than those in the SAP group $(\mathrm{P}<0.05)$. The levels of IL-10 in the RAP group were significantly lower than those in the SAP group $(\mathrm{P}<0.001)$, but observably increased in comparison with the MAP group $(\mathrm{P}<0.001)$. These results suggested that high expression levels of IL-8, IL-10 and TNF- $\alpha$ may be able to distinguish SAP from MAP, but that only IL-10 could differentiate between MAP and RAP.

In Fig. 3D-H, miRNA expression levels were compared among NC, MAP, SAP and RAP groups. The levels of 
A

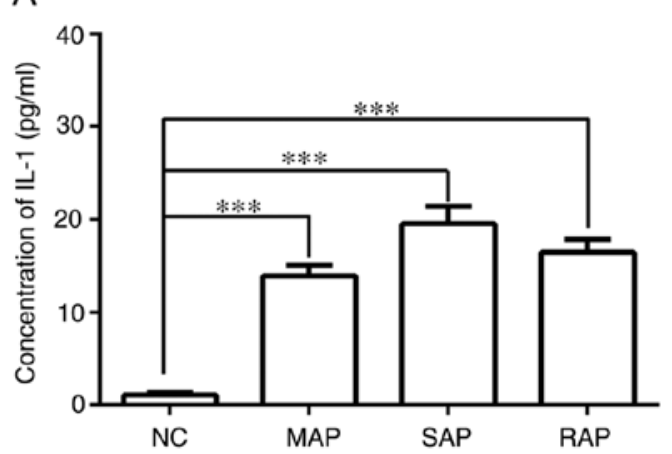

C

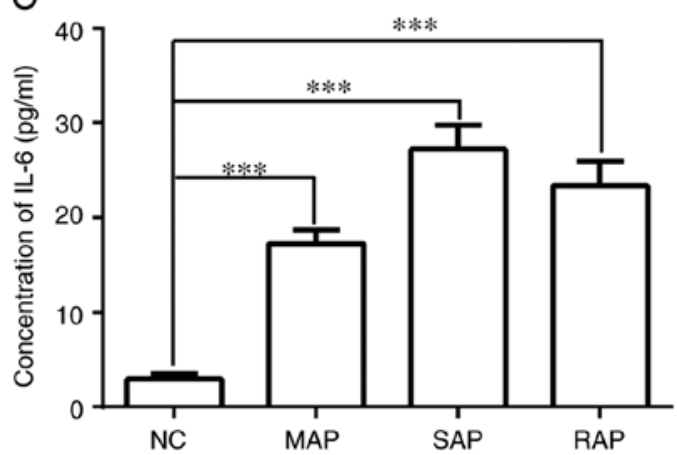

E

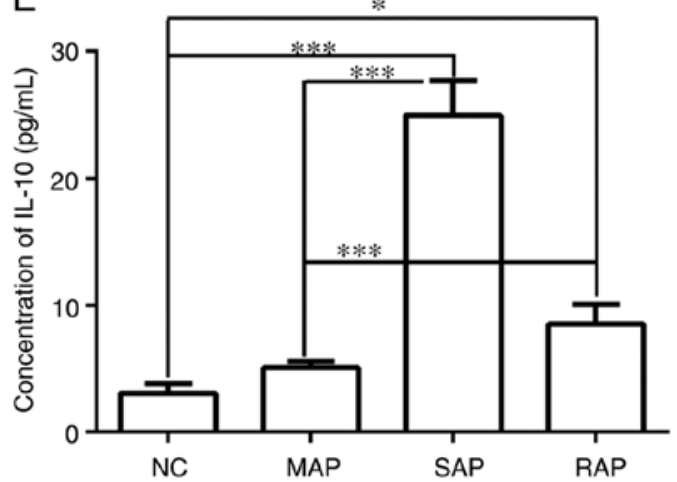

G

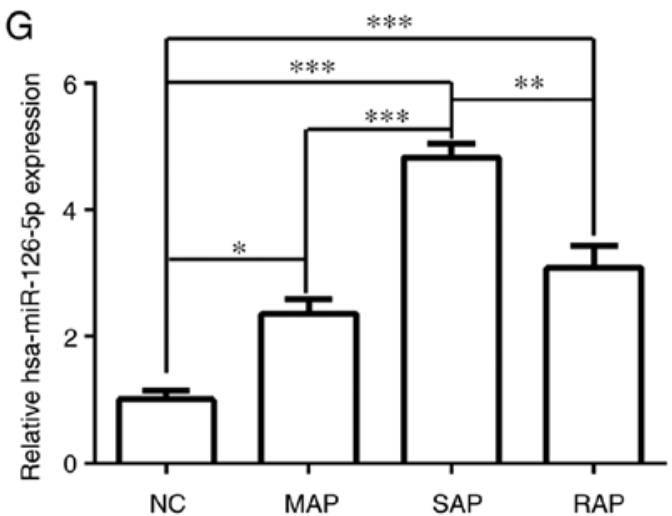

B

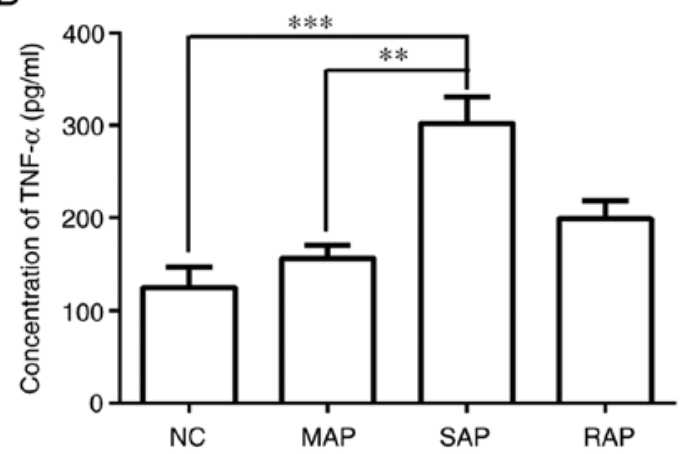

D

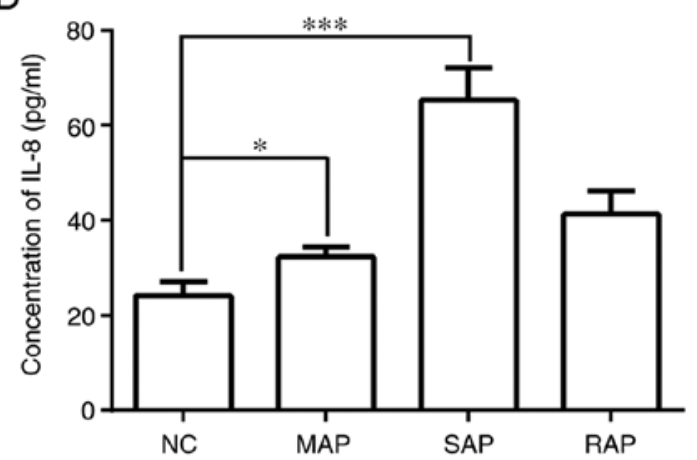

F

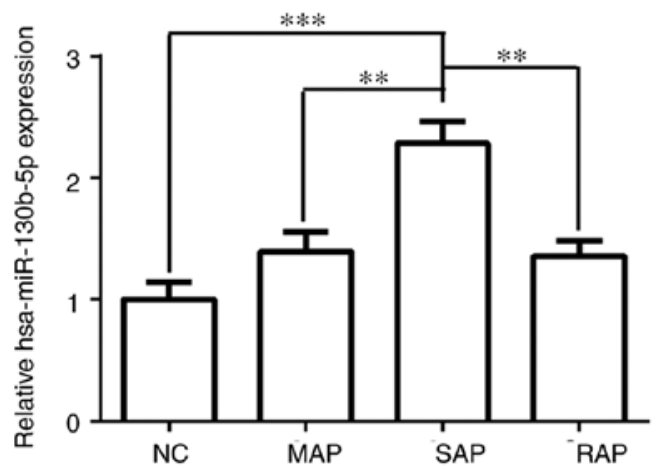

$\mathrm{H}$

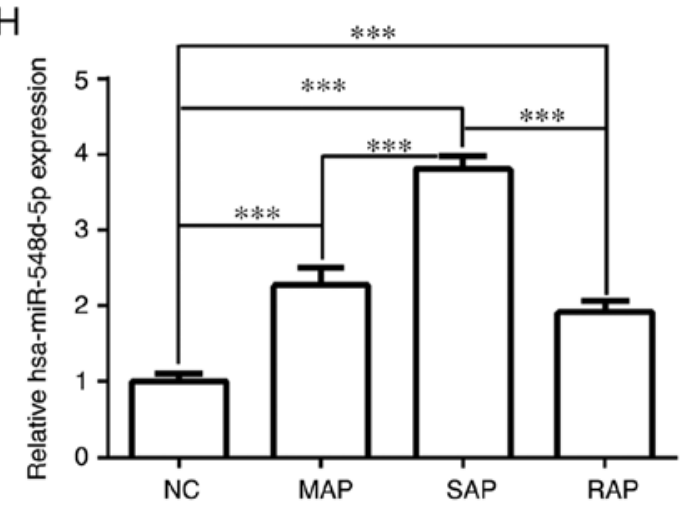

Figure 3. Expression of pro-inflammatory factors and miRNAs in patients with different AP classification. (A) IL-1; (B) TNF- $\alpha$; (C) IL-6; (D) IL-8; (E) IL-10; (F) hsa-miR-130b-5p; (G) hsa-miR-126-5p; (H) hsa-miR-548d-5p. Values are expressed as the mean \pm standard error. One-way analysis of variance with Dunnett's test was used for normally distributed data and the Kruskal-Wallis test with Dunn's post-hoc test for data with a non-normal distribution. ${ }^{*} \mathrm{P}<0.05{ }^{* *} \mathrm{P}<0.01$ and ${ }^{* * *} \mathrm{P}<0.001$. Groups: NC, normal control; MAP, mild AP; SAP, severe AP; RAP, recurrent AP. AP, acute pancreatitis; miR, microRNA; hsa, Homo sapiens.

hsa-miR-126-5p and hsa-miR-548d-5p in the MAP, SAP and RAP groups were significantly higher than those in the NC group $(\mathrm{P}<0.05)$. The expression of hsa-miR-130b-5p was markedly increased in the SAP group in comparison with the NC group $(\mathrm{P}<0.001)$. In addition, the expression levels of hsa-miR-130b-5p, hsa-miR-126-5p and hsa-miR-548d-5p were higher in the SAP group than the MAP and RAP groups. However, no significant difference in the levels of these 
miRNAs was obtained between the MAP and RAP group. These results suggested that high expression levels of miRNAs were able to distinguish SAP from MAP and RAP group but were not able to differentiate between MAP and RAP.

Analysis of candidate miRNAs and pro-inflammatory factors to determine AP classification using single-and multifactor binary logistic regression. Univariate logistic regression analysis was performed with AP as the dependent variable and serum levels of the inflammatory factors and miRNAs as independent variables (Table SII). The results of the single-factor binary logistic regression analysis were consistent with those of the nonparametric Mann-Whitney $U$ test. The serum levels of the following inflammatory factors and miRNAs were risk factors for AP: IL-1 [odds ratio $(\mathrm{OR})=2.311,95 \%$ CI: $1.430-3.734$, $\mathrm{P}=0.001], \mathrm{IL}-6(\mathrm{OR}=2.311,95 \% \mathrm{CI}: 1.430-3.734, \mathrm{P}=0.001), \mathrm{IL}-8$ (OR=1.043, 95\% CI: 1.018-1.070, $\mathrm{P}=0.001), \mathrm{IL}-10(\mathrm{OR}=1.232$, 95\% CI: $1.088-1.395, \mathrm{P}=0.001), \mathrm{TNF}-\alpha(\mathrm{OR}=1.007,95 \% \mathrm{CI}$ : 1.003-1.011, $\mathrm{P}=0.001)$, hsa-miR-126-5p (OR=3.575, 95\% CI: 2.072-6.170, $\mathrm{P}<0.001)$, hsa-miR-130b-5p (OR=2.716, 95\% CI: 1.486-4.963, $\mathrm{P}=0.001)$ and hsa-miR-548d-5p $(\mathrm{OR}=5.848$, 95\% CI: 2.751-12.430, $\mathrm{P}<0.001)$.

The correlation between AP severity and inflammatory factors and miRNA in serum was determined by Spearman correlation analysis, as presented in Fig. 4. The levels of IL-1, IL-6, IL-10, hsa-miR-126-5p and hsa-miR-130b-5p all had strong positive correlations with AP severity (complete correlation: $r=1$; strong correlation: $0.7 \leq r<1$; moderate correlation: $0.4 \leq \mathrm{r}<0.7$; weak correlation: $\mathrm{r}<0.4$; no correlation: $\mathrm{r}=0$.) (19). The levels of IL-8, TNF- $\alpha$ and hsa-miR-548d-5p had moderate positive correlations with AP severity. However, the expression of IL-1, IL-6, hsa-miR-126-5p, and hsa-miR-548d-5p exhibited no statistically significant association with AP when multifactor logistic regression analysis was performed (Table SIII). This result may be due to the existence of confounding factors. However, further multifactorial binary logistic regression analysis (combining these 4 factors freely) indicated that single inflammatory factor (IL-1 or IL-6) and miRNA (hsa-miR-126-5p or hsa-miR-548d-5p) may be used to predict the classification of AP $(\mathrm{P}<0.05)$. According to the results of this analysis, an equation may in future be established to predict the probability of AP. The results of the ROC curve analysis were consistent with the results of the multifactorial binary logistic regression analysis (Table III). The combined detection of IL-1 + hsa-miR-126-5p (AUC, 0.991; sensitivity, $95.6 \%$; specificity, $100 \%$; cutoff value, 0.641; $\mathrm{P}<0.001$ ), IL-6 + hsa-miR-126-5p (AUC, 0.991; sensitivity, 93.3\%; specificity, 96.7\%; cutoff value, 0.802 ; $\mathrm{P}<0.001$ ), IL-1 + hsa-miR-548d-5p (AUC, 0.990; sensitivity, 95.6\%; specificity, $100 \%$; cutoff value, 0.830 ; $\mathrm{P}<0.001)$, and IL-6 + hsa-miR-548d-5p (AUC, 0.980; sensitivity, 92.2\%; specificity, 96.7\%; cutoff value, 0.795 ; $\mathrm{P}<0.001$ ) could distinguish AP from NC. The results of the ROC analysis for serum amylase (AUC, 0.999; sensitivity, 97.8\%; specificity, 100\%; cutoff value, 136.95; $\mathrm{P}<0.001$ ) and lipase (AUC, 1.000; sensitivity, 98.9\%; and specificity, 100\%; cutoff value, 172.75; $\mathrm{P}<0.001)$ showed that they could also differentiate AP from $\mathrm{NC}$. The results indicated that the diagnostic accuracy of IL-1/IL-6 combined with hsa-miR-126-5p/hsa-miR-548d-5p was high but not quite as good as that for amylase and lipase.
Analysis of candidate miRNAs and pro-inflammatory factors by multiclassification disordered logistic regression analysis for AP classification. On the basis of the results of the single- and multifactor binary logistic regression analysis of AP vs. NC and the result of the Spearman correlation analysis (Table SIV), multiclassification disordered logistic regression analysis was performed with AP severity as the dependent variable and the serum levels of the inflammatory factors (IL-1/IL-6) and miRNAs (hsa-miR-126-5p/hsa-miR-548d-5p) as independent variables (Table SV).

Multiclassification disordered logistic regression analysis for AP severity and RAP indicated that the combination of IL-1/IL-6 and hsa-miR-126-5p/hsa-miR-548d-5p had a significant diagnostic value regarding AP types, suggesting that among these factors, IL-1/IL-6 and hsa-miR-126-5p/hsa-miR-548d-5p may be major factors in determining of AP classification $(\mathrm{P}<0.05)$. According to the results of the present analysis, with a greater amount of available data in future an equation may be established to determine the degree of AP severity. Based on the above results, to further evaluate the predictive effect of miRNAs combined with pro-inflammatory cytokines on the severity of AP, ROC curves were drawn and their parameters are presented in Table III. When IL-6 was combined with hsa-miR-126-5p, the AUC (0.952), sensitivity (93.3\%) and specificity (90.0\%) were all the highest for distinguishing MAP from SAP, and the values were also highest for distinguishing MAP from RAP. The predictive effect of IL-1/IL- 6 combined with hsa-miR-126-5p ranked second in the differentiation between SAP and RAP, and the predictive effect of IL-1 or IL-6 combined with hsa-miR-548d-5p was the best (AUC, 0.924, sensitivity, 83.3\%; specificity, 93.3\%; $\mathrm{P}<0.001$ ). Although serum amylase and lipase levels showed superiority in their utility in distinguishing AP from NC, they had a low accuracy in enabling discernment of the distinction between MAP, SAP and RAP.. The results of the present study suggested that the combined detection of IL-6 and hsa-miR-126-5p may be able to better distinguish MAP from SAP and RAP than assessment of amylase or lipase.

\section{Discussion}

AP is closely linked to pro-inflammatory cytokines, inflammatory mediators and pancreatic enzymes, which are also associated with other diseases, including systemic inflammatory response syndrome and supplementary anti-inflammatory response syndrome (20). Thus, predicting AP severity at the early stage is important to grasp the clinical condition, take appropriate treatment measures and determine the prognosis (21). Inflammatory factors and miRNAs have predictive value for the severity of pancreatitis. The combined detection of these factors may improve the efficiency of predicting the severity of AP. In the present study, high expression levels of IL-10, TNF- $\alpha$, hsa-miR-126-5p, hsa-miR-548d-5p and hsa-miR-130b-5p were indicated to be associated with SAP. The combined detection of IL-1/IL- 6 and hsa-miR-126-5p/ hsa-miR-548d-5p was able to effectively differentiate between MAP, SAP and RAP. In particular, the combination of IL-6 and hsa-miR-126-5p had the highest sensitivity and specificity, which may be used to determine the severity of AP. 

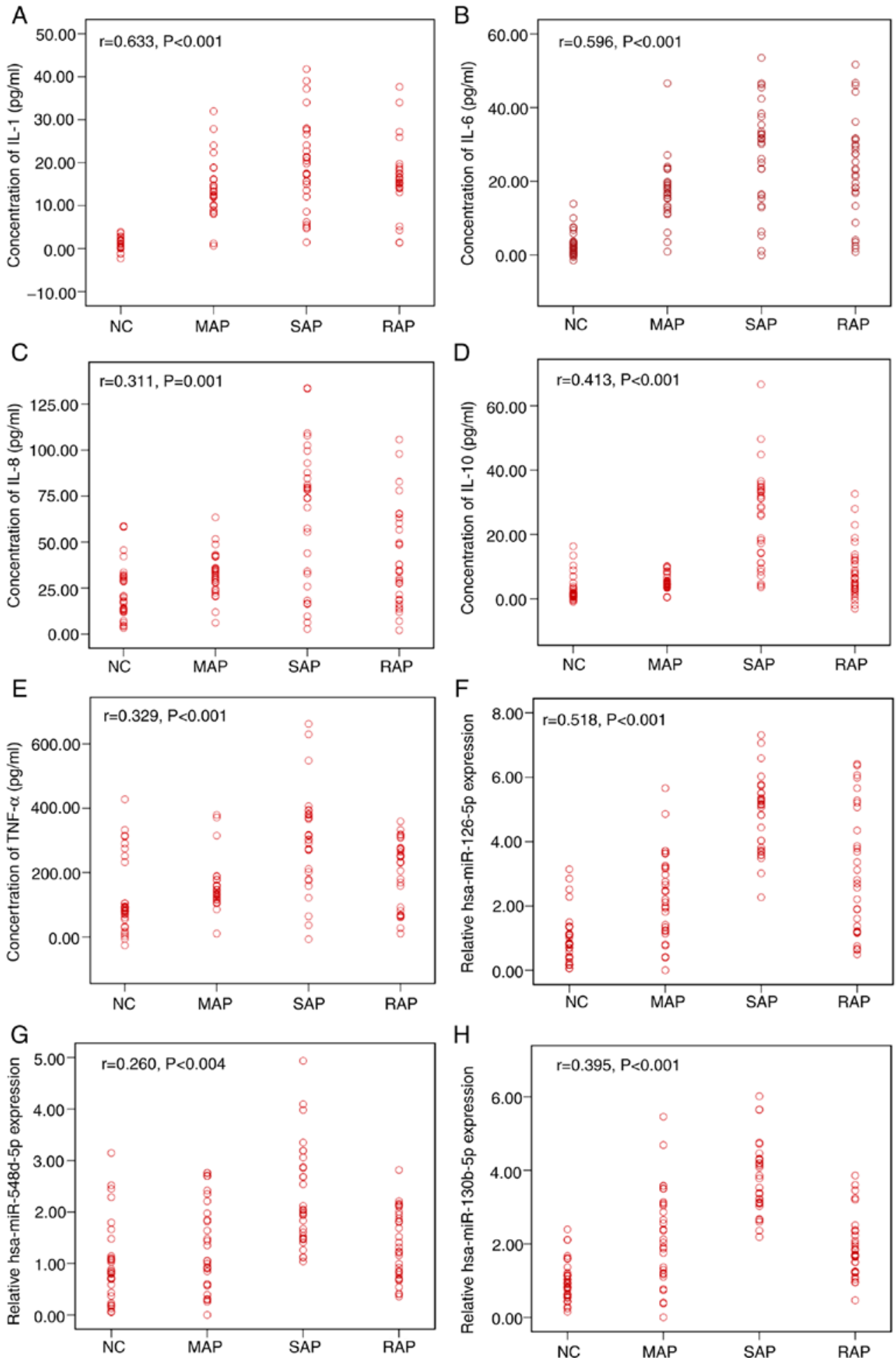

Figure 4. Spearman correlation of AP classification with inflammatory factors and miRNAs. (A) IL-1; (B) IL-6; (C) IL-8; (D) IL-10; (E) TNF- $\alpha$; (F) hsa-miR-126-5p; (G) hsa-miR-548d-5p; (H) hsa-miR-130b-5p. P<0.05 was considered to indicate a significant correlation. Groups: NC, normal control; MAP, mild AP; SAP, severe AP; RAP, recurrent AP. AP, acute pancreatitis; miRNA/miR, microRNA; hsa, Homo sapiens.

Inflammatory mediators of the innate immune response are important in the pathogenesis of AP. IL-4, IL-6, IL-8 and IL-10, which have numerous functions in immune responses and apoptosis in patients with AP, may trigger the defensive response and restrain immune function (22). The severity of AP is associated with increased levels of various pro-inflammatory cytokines, such as IL-6, IL-1 $\beta$ and TNF- $\alpha$, after pancreatic acinar cell injury (23), and the expression of these factors increased with the severity of AP (24). TNF- $\alpha$ is a key initiator of pancreatic inflammation (25), and it stimulates acinar cells to increase the release of IL-6 $(26,27)$. The present study demonstrated that the concentrations of serum TNF- $\alpha$ and IL-8 in the MAP group were all lower than those in the SAP group, which was consistent with the results of Yang et al (5) and suggests that TNF- $\alpha$ and IL- 8 are strongly associated with the severity of AP. IL-6, which has an important role in the 
Table III. Results of the ROC analysis for AP classification.

A, AP vs. NC

\begin{tabular}{|c|c|c|c|c|c|c|c|}
\hline Factors & AUC & SE & $95 \% \mathrm{CI}$ & Sensitivity (\%) & Specificity (\%) & Cutoff value & P-value \\
\hline IL-1+hsa-miR-126-5p & 0.991 & 0.005 & $0.981-1.000$ & 95.6 & 100 & 0.641 & $<0.001$ \\
\hline IL-6+hsa-miR-126-5p & 0.991 & 0.006 & $0.979-1.000$ & 93.3 & 96.7 & 0.802 & $<0.001$ \\
\hline IL-1+hsa-miR-548d-5p & 0.990 & 0.007 & $0.977-1.000$ & 95.6 & 100 & 0.830 & $<0.001$ \\
\hline IL-6+hsa-miR-548d-5p & 0.980 & 0.010 & $0.960-1.000$ & 92.2 & 96.7 & 0.795 & $<0.001$ \\
\hline Lipase & 1.000 & 0.001 & $0.998-1.000$ & 98.9 & 100 & 172.750 & $<0.001$ \\
\hline Amylase & 0.999 & 0.002 & $0.995-1.000$ & 97.8 & 100 & 136.950 & $<0.001$ \\
\hline
\end{tabular}

B, MAP vs. SAP

\begin{tabular}{lccccrrr}
\hline Factors & AUC & SE & 95\% CI & Sensitivity (\%) & Specificity (\%) & Cutoff value & P-value \\
\hline IL-1+hsa-miR-126-5p & 0.926 & 0.036 & $0.856-0.995$ & 90.0 & 86.7 & 0.605 & $<0.001$ \\
IL-6+hsa-miR-126-5p & 0.952 & 0.026 & $0.902-1.000$ & 93.3 & 90.0 & 0.443 & $<0.001$ \\
IL-1+hsa-miR-548d-5p & 0.829 & 0.053 & $0.724-0.933$ & 93.3 & 60.0 & 0.357 & $<0.001$ \\
IL-6+hsa-miR-548d-5p & 0.876 & 0.044 & $0.790-0.962$ & 73.3 & 90.0 & 0.580 & $<0.001$ \\
Lipase & 0.554 & 0.075 & $0.407-0.702$ & 90.0 & 30.0 & 343.000 & 0.469 \\
Amylase & 0.627 & 0.072 & $0.485-0.769$ & 93.3 & 33.3 & 328.450 & 0.090 \\
\hline
\end{tabular}

C, SAP vs. RAP

\begin{tabular}{|c|c|c|c|c|c|c|c|}
\hline Factors & AUC & SE & $95 \% \mathrm{CI}$ & Sensitivity (\%) & Specificity (\%) & Cutoff value & P-value \\
\hline IL-1+hsa-miR-126-5p & 0.754 & 0.066 & $0.626-0.883$ & 60.0 & 93.3 & 0.593 & 0.001 \\
\hline IL-6+hsa-miR-126-5p & 0.754 & 0.066 & $0.626-0.883$ & 60.0 & 93.3 & 0.593 & 0.001 \\
\hline IL-1+hsa-miR-548d-5p & 0.924 & 0.033 & $0.859-0.989$ & 83.3 & 93.3 & 0.632 & $<0.001$ \\
\hline IL-6+hsa-miR-548d-5p & 0.924 & 0.033 & 0.859-0.989 & 83.3 & 93.3 & 0.632 & $<0.001$ \\
\hline Lipase & 0.542 & 0.075 & $0.395-0.690$ & 53.3 & 63.3 & 489.650 & 0.574 \\
\hline Amylase & 0.409 & 0.074 & $0.264-0.554$ & 100.0 & 3.3 & 132.300 & 0.225 \\
\hline
\end{tabular}

D, MAP vs. RAP

\begin{tabular}{|c|c|c|c|c|c|c|c|}
\hline Factors & AUC & SE & $95 \% \mathrm{CI}$ & Sensitivity (\%) & Specificity (\%) & Cutoff value & P-value \\
\hline IL-1+hsa-miR-126-5p & 0.926 & 0.036 & $0.856-0.995$ & 90.0 & 86.7 & 0.544 & $<0.001$ \\
\hline IL-6+hsa-miR-126-5p & 0.952 & 0.026 & $0.902-1.000$ & 93.3 & 90.0 & 0.443 & $<0.001$ \\
\hline IL-1+hsa-miR-548d-5p & 0.829 & 0.053 & $0.724-0.933$ & 93.3 & 60.0 & 0.358 & $<0.001$ \\
\hline IL-6+hsa-miR-548d-5p & 0.876 & 0.044 & $0.790-0.962$ & 73.3 & 90.0 & 0.580 & $<0.001$ \\
\hline Lipase & 0.580 & 0.074 & $0.434-0.726$ & 66.7 & 50.0 & 441.450 & 0.287 \\
\hline Amylase & 0.559 & 0.075 & $0.412-0.706$ & 33.3 & 86.7 & 526.600 & 0.433 \\
\hline
\end{tabular}

NC, normal control; AP, acute pancreatitis; SAP, severe AP; MAP, mild AP; RAP, recurrent AP; AUC, area under the ROC curve; SE, standard error; ROC, receiver operating characteristic.

pathogenesis of the disease, may be used as an early predictive marker for AP severity, and its expression level is positively correlated with AP severity $(28,29)$. Although the serum level of IL-6 was higher in the SAP group than in the MAP and RAP groups, there was no significant difference among these three groups in the present study. The inconsistencies among earlier studies may be due to differences in the populations evaluated, sample sizes and the selection of patients/controls. The rs1800896 and 1082A/G polymorphisms of the IL-10 gene have been identified to contribute to the process of AP development $(30,31)$. In the present study, the level of IL-10 was higher in the SAP group than in the MAP and RAP groups, which also indicated that IL-10 is associated with AP severity. Pro-inflammatory cytokines may result in the 
incidence of compensatory anti-inflammatory response syndrome in the early stage of AP, and they are able to interact with one another to magnify the inflammatory response to expedite the progression of renal failure (32). The expression of the pro-inflammatory cytokines TNF- $\alpha$, IL-1 $\beta$ and IL- 6 was inhibited to suppress the inflammatory response $(33,34)$. The expression levels of inflammatory factors contribute to the severity of AP.

The stable expression of miRNAs in peripheral blood makes their detection convenient and has important prospects in the early diagnosis and treatment of AP (35). In the pathogenesis of AP, a series of changes occur in the expression profile of pancreatic tissue proteins. During this process, miRNAs have an important role in gene expression regulation. With increasing research efforts, more ideal markers of AP may be identified among serum miRNAs, which will help to improve the diagnosis of AP, the therapeutic effect and the prognosis of pancreatitis. A previous integrated transcriptome analysis determined that hsa-miR-130b was highly relevant to pancreatic cancer (36), and microarray data on chronic pancreatitis (37) and pancreatic cancer tissues (52 pairs) indicated that its expression is significantly reduced (12). In the present study, the serum levels of hsa-miR-130b-5p were higher in the SAP group than in the other groups. These differences may be due to differences in the selection and the size of the sample. According to a previous ROC analysis, serum hsa-miR-216a-5p may be used to distinguish MAP from SAP (10). The present study suggested that the level of hsa-miR-216a-5p was higher in the SAP group than in the other groups, but there was no significant difference between the MAP and RAP groups. Other factors may be studied to predict the severity of AP. Hsa-miR-548d overexpression in the PANC-1 human pancreatic carcinoma cell line resulted in impaired cell proliferation (11), suggesting that hsa-miR-548d may be considered a marker for AP. The trend of hsa-miR-548d-5p in RAP and other groups was similar to that of hsa-miR-216a-5p.

Although the present study provided useful results, certain limitations should be considered. First, the sample size of the present study was small, and further studies with large sample sizes are required to validate the present conclusions. Furthermore, no bioinformatics or molecular biology studies were performed to investigate the association between inflammatory cytokines and miRNAs. In addition, cytokines and miRNAs were studied only once during the onset of disease, while dynamic monitoring may be better to assess their predictive value for AP severity. Lastly, the study did not provide clear values for the combined detection of IL-1/IL-6 and hsa-miR-126-5p/hsa-miR-548d-5p to distinguish AP from NC. These will be examined in future studies.

In conclusion, the present results suggest that the detection of miRNAs combined with inflammatory cytokines may be useful in predicting AP severity. In addition, the present study indicated that, compared with the other combinations assessed, IL-6 combined with hsa-miR-126-5p had the highest sensitivity and specificity for the stratification of AP.

\section{Acknowledgements}

Not applicable.

\section{Funding}

The present study was supported by Fujian Traditional Chinese Medicine Science and Technology Project in 2016-2019 [document no. Fujian-Wei TCM Letter (2017) 237, grant no. 2017FJZYLC105] and the Research Subsidy Project of Jinjiang Hospital of Traditional Chinese Medicine Affiliated to Fujian University of Traditional Chinese Medicine in 2017 (grant no. YN201703).

\section{Availability of data and materials}

The datasets used and/or analyzed during the current study are available from the corresponding author on reasonable request.

\section{Authors' contributions}

YJC and TLL made substantial contributions to the conception and design of the study. YJC, TLL, ZC, CHY, SRG and YDZ made substantial contributions to the acquisition, analysis and interpretation of data. YJC participated in drafting the manuscript, and TLL and ZC revised it critically for important intellectual content. All authors read and approved the final version to be published and agreed to be accountable for all aspects of the work in ensuring that questions related to the accuracy or integrity of any part of the work are appropriately investigated and resolved.

\section{Ethics approval and consent to participate}

The study was approved by the Ethics Committee of Jinjiang Hospital of Traditional Chinese Medicine (Jinjiang, China). Written informed consent was obtained from all the subjects.

\section{Patient consent for publication}

Not applicable.

\section{Competing interests}

The authors declare that they have no competing interests.

\section{References}

1. Banks PA, Bollen TL, Dervenis C, Gooszen HG, Johnson CD, Sarr MG, Tsiotos GG and Vege SS; Acute Pancreatitis Classification Working Group: Classification of acute pancreatitis - 2012: Revision of the Atlanta classification and definitions by international consensus. Gut 62: 102-111, 2013.

2. Tenner S, Baillie J, DeWitt J and Vege SS: ACoG. American College of Gastroenterology guideline: Management of acute pancreatitis. Am J Gastroenterol 109: 1400-1415, 2014.

3. Koutroumpakis E, Slivka A, Furlan A, Dasyam AK, Dudekula A, Greer JB, Whitcomb DC, Yadav D and Papachristou GI: Management and outcomes of acute pancreatitis patients over the last decade: A US tertiary-center experience. Pancreatology 17: 32-40, 2017.

4. Żorniak M, Beyer G and Mayerle J: Risk stratification and early conservative treatment of acute pancreatitis. Visc Med 35: 82-89, 2019.

5. Yang YZ, Xiang Y, Chen M, Xian LN and Deng XY: Clinical significance of dynamic detection for serum levels of MCP-1, TNF- $\alpha$ and IL-8 in patients with acute pancreatitis. Asian Pac J Trop Med 9: 1111-1114, 2016. 
6. Vasseur P, Devaure I, Sellier J, Delwail A, Chagneau-Derrode C, Charier F, Tougeron D, Tasu JP, Rabeony H, Lecron JC, et al High plasma levels of the pro-inflammatory cytokine IL-22 and the anti-inflammatory cytokines IL-10 and IL-1ra in acute pancreatitis. Pancreatology 14: 465-469, 2014.

7. Ceranić DB, Zorman M and Skok P: Interleukins and inflammatory markers are useful in predicting the severity of acute pancreatitis. Bosn J Basic Med Sci 20: 99-105, 2020.

8. Qin X, Yan L, Zhao X, Li C and Fu Y: microRNA-21 overexpression contributes to cell proliferation by targeting PTEN in endometrioid endometrial cancer. Oncol Lett 4: 1290-1296, 2012.

9. Cherie B, Askelund KJ, Shanbhag ST, Mandira C, Petrov MS, Brett D, et al: MicroRNAs in mesenteric lymph and serum during acute pancreatitis. Pancreatology 14: S57-S57, 2014.

10. Kuśnierz-Cabala B, Nowak E, Sporek M, Kowalik A Kuźniewski M, Enguita FJ and Stępień E: Serum levels of unique miR-551-5p and endothelial-specific miR-126a-5p allow discrimination of patients in the early phase of acute pancreatitis. Pancreatology 15: 344-351, 2015.

11. Heyn H, Schreek S, Buurman R, Focken T, Schlegelberger B and Beger C: MicroRNA miR-548d is a superior regulator in pancreatic cancer. Pancreas 41: 218-221, 2012.

12. Zhao G, Zhang JG, Shi Y, Qin Q, Liu Y, Wang B, Tian K, Deng SC, Li X, Zhu S, et al: MiR-130b is a prognostic marker and inhibits cell proliferation and invasion in pancreatic cancer through targeting STAT3. PLoS One 8: e73803, 2013.

13. Goyal A, Jain M, Rehberg K, Goodman W and Gertner E: Pancreatic panniculitis in active systemic lupus erythematosus. J Cutan Pathol 46: 688-690, 2019.

14. Xu YW, Li R and Xu SC: Hypothyroidism with elevated pancreatic amylase and lipase without clinical symptoms: A case report. World J Clin Cases 8: 3299-3304, 2020.

15. Balthazar EJ, Robinson DL, Megibow AJ and Ranson JH: Acute pancreatitis: Value of CT in establishing prognosis. Radiology 174 331-336, 1990.

16. Cholette JM,Pietropaoli AP,Henrichs KF, Alfieris GM, Powers KS, Gensini F, Rubenstein JS, Sweeney D, Phipps R, Spinelli SL, et al Elevated free hemoglobin and decreased haptoglobin levels are associated with adverse clinical outcomes, unfavorable physiologic measures, and altered inflammatory markers in pediatric cardiac surgery patients. Transfusion 58: 1631-1639, 2018.

17. Blondal T, Jensby Nielsen S, Baker A, Andreasen D, Mouritzen P, Wrang Teilum $M$ and Dahlsveen IK: Assessing sample and miRNA profile quality in serum and plasma or other biofluids. Methods 59: S1-S6, 2013.

18. Maruyama T, Nishihara K, Umikawa M, Arasaki A, Nakasone T, Nimura F, Matayoshi A, Takei K, Nakachi S, Kariya KI, et al MicroRNA-196a-5p is a potential prognostic marker of delayed lymph node metastasis in early-stage tongue squamous cell carcinoma. Oncol Lett 15: 2349-2363, 2018.

19. Hashmi A, Cahill GL, Zaldana M, Davis G, Cronin BJ, Brandel MG, et al: Can head circumference be used as a proxy for intracranial volume in patients with craniosynostosis? Ann Plast Surg 82 (Suppl 4): S295-S300, 2019.

20. Yang CJ, Chen J, Phillips ARJ, Windsor JA and Petrov MS Predictors of severe and critical acute pancreatitis: A systematic review. Dig Liver Dis 46: 446-451, 2014.

21. Dumnicka P,Kuśnierz-Cabala B, SporekM,Mazur-LaskowskaM, Gil K, Kuźniewski M, Ceranowicz P, Warzecha Z, Dembiński A, Bonior J, et al: Serum concentrations of angiopoietin-2 and soluble fms-like tyrosine kinase 1 (sFlt-1) are associated with coagulopathy among patients with acute pancreatitis. Int J Mol Sci 18: e753, 2017.
22. Reihmane D and Dela F: Interleukin-6: Possible biological roles during exercise. Eur J Sport Sci 14: 242-250, 2014.

23. Qiu L, Sun RQ, Jia RR, Ma XY, Cheng L, Tang MC and Zhao Y: Comparison of existing clinical scoring systems in predicting severity and prognoses of hyperlipidemic acute pancreatitis in Chinese patients: A retrospective study. Medicine (Baltimore) 94 : e957, 2015

24. Sternby H, Hartman H, Thorlacius $H$ and Regnér S: IL-1 $\beta$, IL-6, IL-8 and IL-10 are important chronological biomarkers in the inflammatory development of acute pancreatitis. Pancreatology 18: S100, 2018.

25. Rae D, Bowyer RC and Wharton RQ: Inflammatory mediators in acute pancreatitis. Br J Surg 82: 855, 1995.

26. Chi DZ, Chen J and Huang DP: Influence of interleukin-1 $\beta$ and interleukin-6 gene polymorphisms on the development of acute pancreatitis. Genet Mol Res 14: 975-980, 2015.

27. Park J, Chang JH, Park SH, Lee HJ, Lim YS, Kim TH, Kim CW and Han SW: Interleukin-6 is associated with obesity, central fat distribution, and disease severity in patients with acute pancreatitis. Pancreatology 15: 59-63, 2015.

28. Rao SA and Kunte AR: Interleukin-6: An early predictive marker for severity of acute pancreatitis. Indian J Crit Care Med 21: 424-428, 2017

29. Dambrauskas Z, Giese N, Gulbinas A, Giese T, Berberat PO, Pundzius J, Barauskas G and Friess H: Different profiles of cytokine expression during mild and severe acute pancreatitis. World J Gastroenterol 16: 1845-1853, 2010.

30. Zhou H, Liu A, Zhou B, Zhao C and Jin G: Interleukin-10 gene rs1800896 polymorphism increases risk of acute pancreatitis. Medicine (Baltimore) 96: e9006, 2017.

31. Jiang BZ, Tang L, Xue H and Liu DP: Role of IL-10 gene polymorphisms in the development of acute pancreatitis. Genet Mol Res 15: 15027743, 2016.

32. Bao XB, Ma Z, Gu JB, Wang XQ, Li HG and Wang WY: IL-8 $-251 \mathrm{~T} / \mathrm{A}$ polymorphism is associated with susceptibility to acute pancreatitis. Genet Mol Res 14: 1508-1514, 2015.

33. Wang $\mathrm{H}$, Zhang Y, Bai R, Wang $\mathrm{M}$ and Du S: Baicalin attenuates alcoholic liver injury through modulation of hepatic oxidative stress, inflammation and sonic hedgehog pathway in rats. Cell Physiol Biochem 39: 1129-1140, 2016.

34. Ouziel R, Gustot T, Moreno C, Arvanitakis M, Degré D, Trépo E, Quertinmont E, Vercruysse V, Demetter P, Le Moine O, et al: The ST2 pathway is involved in acute pancreatitis: A translational study in humans and mice. Am J Pathol 180: 2330-2339, 2012.

35. Zhang J, Ren J, Chen H and Geng Q: Inflammation induced-endothelial cells release angiogenesis associated-microRNAs into circulation by microparticles. Chin Med J (Engl) 127: 2212-2217, 2014.

36. Yang $J$ and Zeng Y: Identification of miRNA-mRNA crosstalk in pancreatic cancer by integrating transcriptome analysis. Eur Rev Med Pharmacol Sci 19: 825-834, 2015.

37. Ofori JK, Salunkhe VA, Bagge A, Vishnu N, Nagao M, Mulder H, Wollheim CB, Eliasson L and Esguerra JL: Elevated $\mathrm{miR}-130 \mathrm{a} / \mathrm{miR} 130 \mathrm{~b} / \mathrm{miR}-152$ expression reduces intracellular ATP levels in the pancreatic beta cell. Sci Rep 7: 44986, 2017.

This work is licensed under a Creative Commons Attribution-NonCommercial-NoDerivatives 4.0 International (CC BY-NC-ND 4.0) License. 